\title{
An Ultrasound-Guided Organ Biopsy Simulation with 6DOF Haptic Feedback
}

\author{
Dong $\mathrm{Ni}^{1}$, Wing-Yin $\mathrm{Chan}^{1}$, Jing Qin ${ }^{1}$, Yingge $\mathrm{Qu}^{1}$, Yim-Pan Chui ${ }^{1}$, \\ Simon S.M. Ho ${ }^{2}$, and Pheng-Ann Heng ${ }^{1}$ \\ ${ }^{1}$ Department of Computer Science and Engineering, \\ The Chinese University of Hong Kong, Hong Kong SAR, China \\ ${ }^{2}$ Union Hospital, Hong Kong SAR, China
}

\begin{abstract}
Ultrasound-guided biopsy is one of the most fundamental, but difficult, skills to acquire in interventional radiology. Intensive training, especially in the needle insertion, is required for trainee radiologists to perform safe procedures. In this paper, we propose a virtual reality simulation system to facilitate the training of radiologists and physicians in this procedures. Key issues addressed include a 3D anatomical model reconstruction, data fusion of multiple ultrasound volumes and computed tomography (CT), realistic rendering, interactive navigation, and haptic feedbacks in six degrees of freedom (DOF). Simulated ultrasound imagery based on real ultrasound data is presented to users, in real-time, while performing an examination on the needle placement into a virtual anatomical model. Our system delivers a realistic haptic feeling for trainees throughout the simulated needle insertion procedure, permitting repeated practices with no danger to patients.
\end{abstract}

\section{Introduction}

Ultrasound-guided biopsy is performed to find an abnormal area of tissue and guide its removal for examination. The success of this procedure is dependant on the correct alignment of the biopsy needle with the scanning plane of the ultrasound probe and with the target lesion, which requires considerable training and practice to perfect. Both visual and haptic feedback are crucial to acquire the hand-eye coordination skills needed in the procedure. Traditionally the training can only be performed on live patients. However, training in patients not only jeopardizes their health, but also provides limited access to training scenarios, and makes it difficult for training in a time efficient manner.

Recently, some work has been devoted to developing simulation system of ultrasound-guided needle insertion procedures. Franck et al. present a simulator for needle guidance training within a virtual environment 11. The haptic model integrated into this system allows skin penetration. However, the realism of the simulated ultrasound images cannot be guaranteed since these images are produced by moving an ultrasound scanner on a foam filled box. Magee et al. [2] introduced an augmented-reality system for ultrasound guided needle placement training. Although this system utilizes a number of techniques to improve the

D. Metaxas et al. (Eds.): MICCAI 2008, Part II, LNCS 5242, pp. 551 559 2008.

(C) Springer-Verlag Berlin Heidelberg 2008 


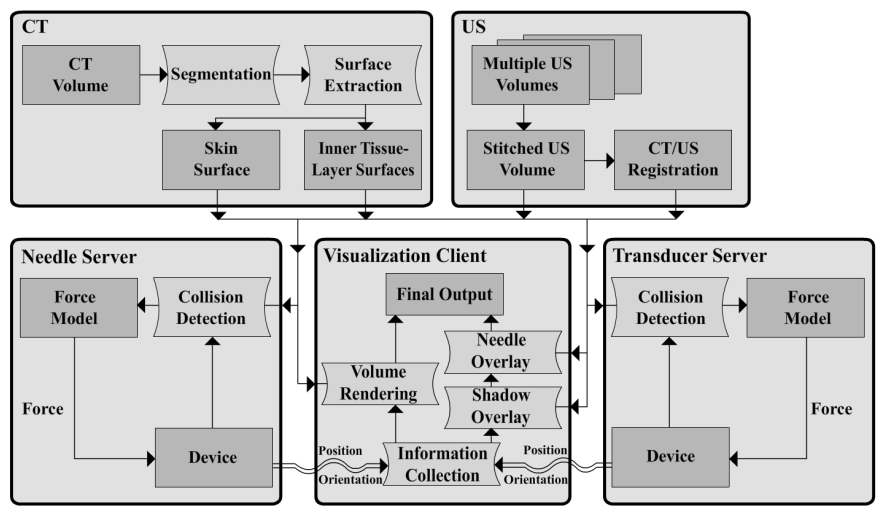

Fig. 1. Overall framework

realism of the simulated ultrasound images, one of the limitations in the system is that it cannot provide realistic force sensation. Forest et al. [3] presented a simulator with haptic devices for ultrasound examination and ultrasound guided needle insertion. The general appearance of the simulated ultrasound images is mainly based on CT or MRI images, which may differs from real ultrasound. Meanwhile, the needle is simulated by adopting a 3DOF force feedback device which cannot provide resistance torque force to maintain the original path of needle insertion.

In this paper, we propose a virtual reality based simulation system for training ultrasound-guided needle insertion with realistic visual and haptic feedback. Currently, we focus on the liver region. The overall framework of our system is shown in Fig. 1. Multiple ultrasound volumes with different scan angles are first stitched to generate an ultrasound panorama, which is then registered with CT to obtain a correlation between CT and the ultrasound volume. Anatomic surfaces are extracted from CT for both visual and haptic rendering while the stitched ultrasound volume is for simulating the ultrasound imagery. To provide a capability of two-hands training, we make use of two haptic devices to simulate a transducer and a needle. We adopt a client-server architecture to parallelize visualization and haptic rendering in order to minimize performance overhead occuring in concurrently running haptic and visual rendering processes on a same machine. The visualization client is responsible for (1) collecting transformation from the haptic devices, (2) rendering simulated ultrasound imagery, given by the stitched ultrasound volume, with an overlay of shadows and needle, and (3) rendering an anatomical exploration view showing the virtual transducer/needle and virtual organs. Simultaneously, the two haptic servers can perform collision detection and haptic rendering at a relatively higher update rate. This paper is organized as follows. Ultrasound data processing and stitching is introduced in section 2. Visual and haptic rendering will be described in section 3 and 4 respectively. Finally, results and discussion are given in section 5 . 


\section{Ultrasound Data Processing and Stitching}

To enlarge the field-of-view of ultrasound images, multiple ultrasound volumes are first co-registered and stitched to create a ultrasound panorama. ScaleInvariant Feature Transform (SIFT) [4 has been proved to be successful in extracting distinctive invariant features from 2D images for registration. Based on [5], we adopt a modified 3D-SIFT descriptor to register and stitch multiple ultrasound volumes. We first pre-process the ultrasound data and detect 3D keypoints from multiple ultrasound volumes. A 3D feature descriptor is then constructed for ultrasound stitching.

Ultrasound Pre-processing. An inherent characteristic of ultrasound imaging is the presence of shadows, which may hinder the detection and matching of keypoints. Therefore, we have to mask them before registration. Shadow is mainly caused by a loss of signal along the direction of the beam due to strong acoustic reflection. To detect shadows, we first determine a threshold value based on experimental ultrasound (US) scans in advance. The US image is scanned along the echo direction from the bottom to the transducer surface; along the beam, we label all consecutive pixels with intensity value lower than the predefined threshold as shadows. The search terminates once a value higher than the threshold is found. Shadow regions shall not be processed in subsequent steps.

Detection of 3D Keypoints. Keypoint candidates are first found using the difference-of-Gaussian (DOG) scale-space extrema 4]. Each sample point is compared to its 26 neighbors in the current volume and 27 neighbors in the volumes of scale above and below. It is selected as a keypoint candidate only if its intensity is larger than those of all its neighbors or smaller than those of all of them. After all keypoint candidates are found, the next step is to eliminate candidates that have poor contrast or poorly localized along an edge. Edge responses (in 3D) can be measured by a $3 \times 3$ Hessian matrix, $H$, which describes the curvatures at the keypoint. A poor candidate usually presents a large principal curvature across the edge but a small one in the perpendicular direction. Given an ordered eigenvalues of $H\left(\left|\lambda_{1}\right|<\left|\lambda_{2}\right|<\left|\lambda_{3}\right|\right)$ with corresponding eigenvectors $\left(e_{1}, e_{2}, e_{3}\right)$, the eigenvectors define an orthogonal coordinate system aligned with the direction of minimal $\left(e_{1}\right)$ and maximal $\left(e_{3}\right)$ curvature. Then we refer a keypoint to be valid under the constraint: $r=\frac{|\lambda 3|}{\lambda 1 \mid}<T$. T is commonly chosen as 10 in image registration. Since ultrasound images are more noisy and not as sharp as normal images, we lessen this restriction to $T=15$ in order to retain more keypoint candidates.

Construction of 3D SIFT Descriptor. Each keypoint is assigned a dominant orientation based on local image properties so that the keypoint descriptor can be represented relative to this orientation ( $\theta$ denotes the longitude while $\phi$ denotes the latitude) [5]. To compute the SIFT descriptor, first, the 3D neighborhood surrounding the keypoint is rotated so that the dominant orientation points to the direction of $\theta=\phi=0$. Sub-histograms are then constructed by sampling the sub-regions surrounding the keypoint. Each SIFT descriptor is created from 
$64(4 \times 4 \times 4)$ sub-regions, where each sub-region includes $64(4 \times 4 \times 4)$ pixels. In our case, we adopt 8 bins (of $360^{\circ}$ ) for $\theta$ and 4 bins (of $180^{\circ}$ ) for $\phi$, where each bin for the gradient orientation is 45 degrees wide. The two orientation angles should be recalculated in the rotated region. Finally, a $4 \times 4 \times 4 \times 8 \times 4=2048$ element feature vector for each keypoint is used.

Stitching of Multiple Ultrasound Volumes. Given two ultrasound volumes (A and B), the keypoints in the two volumes are obtained respectively. For one keypoint in volume A, the Euclidean distances between it and all keypoints in volume $\mathrm{B}$ are calculated. Then by comparing the ratio of distance of the closest neighbor to that of the second-closest neighbor, all matches in which the distance ratio is greater than a threshold is rejected. Since we are working on a single modality (ultrasound) registration, the intensity difference between correctly matched keypoint pair should be not very large. Therefore, we also eliminate false matching pairs by comparing the intensity difference between the keypoint pair. The comparison is performed by using the histogram distance of greylevels inside an $8 \times 8$ neighbor around the keypoint. Finally we employ the RANSAC algorithm [6] to simultaneously solve the correspondence problem and estimate the transformation matrix related to such a pair of volumes. Once pairwise matches have been established between the volumes, we can obtain a global transformation matrix for each volume and stitch them into a panorama.

\section{Visual Rendering}

In order to provide closely-coupled anatomical visualization to the simulated ultrasonic view, we correlate the CT with the stitched ultrasound volume (Fig.2(a)). Landmark pairs are first manually selected by experienced radiologists from the $\mathrm{CT}$ and US volume and they are used as the initialization of the registration process; mutual information is then used to optimize the result.

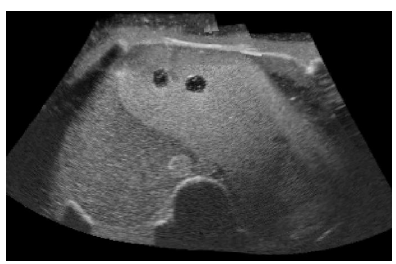

(a)

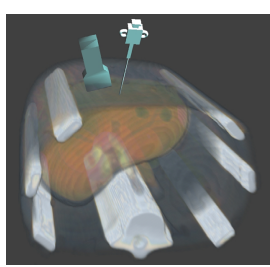

(b)

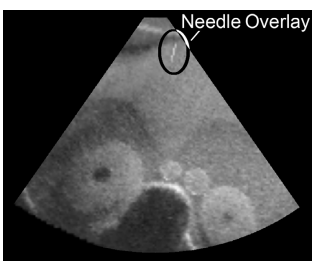

(c)

Fig. 2. (a) Stitched volume, (b) anatomical navigation, and (c) the simlation

As shown in Fig. 2(b), the CT volume is volume rendered and depicted in a navigation view together with the virtual surgical instruments like transducer and biopsy needle. The CT data also provides extra information e.g. bone for simulating the visual artifact commonly observed in ultrasound imagery, shadows, 
in the simulated ultrasound view. Shadows are generated through ray-casting. A rendering of needle shaft is overlayed on top of the stitched ultrasound volume for training biopsy procedure (Fig. [2(c)).

\section{Haptic Rendering}

We use one SensAble PHANTOM Omni and one PHANTOM Premium 1.5 High Force/6DOF to simulate the transducer and the needle (Fig 3(a)), respectively. The latter device can provide 6DOF force feedback, which is necessary to maintain the needle trajectory after puncturing skin. We adopt different schemes to simulate the feel of transducer and needle manipulations. For the transducer, we set a constraint on the skin surface to restrict the virtual transducer from passing through it. User can feel a resistance force when the virtual transducer collides with the skin. Force modeling for needle insertion is more complicated since force components from different sources should be modelled differently.

\subsection{Modeling of Needle Insertion Force}

In order to accurately model the feedback forces, it is necessary to characterize the typical force profile of needle insertion from skin into liver. Based on biomechanical literature [7, there are three force peaks in the force-displacement relationship during the procedure, indicating the puncture of skin, muscle and liver's capsule. In each peak, there is a rise of force resulted from the stiffness of tissues, followed by a sudden drop of force during puncture. After every puncture event, the force fluctuates with a slightly increasing trend, due to the increasing friction resulted from the graduate increase in contact area between the needle and underlying tissues. When the needle penetrates into the liver, we consider forces caused by cutting liver internal structures such as arteries or veins. Based on the needle force transients, we implement three models to simulate the pre-puncture force, friction force and cutting force, respectively. In addition, after puncturing the skin, path constraint force and torque are simulated to prevent the virtual needle from deviating the original trajectory. Finally, we compute the total force and torque in every haptic frame by adding them all.

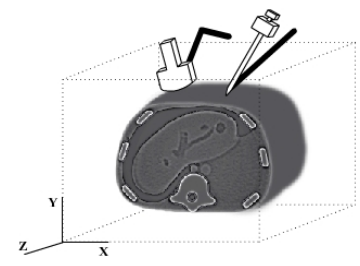

(a)

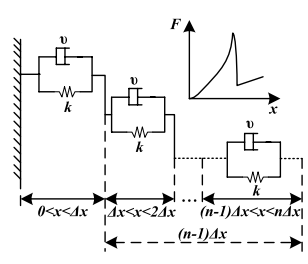

(b)

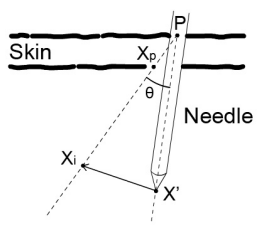

(c)

Fig. 3. Haptic rendering: (a) the haptic devices setup, (b) incremental viscoelastic model, and (c) path constraint force/torque modeling 
Pre-puncture force Modeling. We employed the incremental viscoelastic model developed by Brett et al. [7] to model the pre-puncture forces of skin, muscle and liver's capsule. The principle of the model is shown in Fig. 3(b). It starts with a single Voigt element, a new Voigt element is added each time whenever the total needle displacement $x$ exceeds a multiple of an adjustable variable, $\Delta x$. The force required to make a displacement of $x$ before puncture is:

$$
F_{\text {pre-puncture }}(x, v)=\frac{x\left(6 \nu v \Delta x+k x^{2}+3 k x \Delta x+2 k \Delta x^{2}\right)}{6 \Delta x^{2}}
$$

where $k$ and $\nu$ are the elastic coefficient and viscous coefficient of the tissue while $v$ is the velocity of the needle.

Friction Force Modeling. The friction forces occur along the needle insertion path and caused by the relative motion between the needle and tissues. Due to the needle clumping, there exists fluctuation of friction forces during the penetration procedure. To simulate this feel, we add a random quantity generated equally from the range of $[-\delta \mu, \delta \mu]$ to the friction forces, where $\mu$ is the coefficient of kinetic friction and $\delta$ is a variable which can be adjusted according to the roughness of tissues. Thus, the friction forces can be calculated by:

$$
F_{\text {friction }}(x, v)=-v \nu *(2 \pi r x)+\operatorname{random}(-\delta \mu, \delta \mu)
$$

where $v$ and $r$ is the velocity and radius of the needle; $\nu$ is the viscosity of related tissue and $x$ is the displacement of needle penetration into the related tissue.

Cutting Force Modeling. The cutting force is caused by the collisions with and puncture of the interior structures of liver. According to [8], cutting force can be considered as a constant force $F_{c}$. In the skin, adipose tissues and muscle, we neglect cutting force since they don't contain such a substantial number of arteries and veins as liver.

Needle Path Constraint. To maintain the insertion path, our system provides force feedback and torque feedback to avoid users from translating or rotating the penetrated needle. The principles of force feedback and torque feedback are shown in Fig 3(c), where $P$ is the contact point of skin surface and $X_{p}$ is the puncture point of skin. Therefore the insertion path can be determined by vector $\boldsymbol{P} \boldsymbol{X}_{\boldsymbol{p}}$. At some moment, if the stylus tip deviates the original path from $X_{i}$ to $X^{\prime}$, the device will generate a residence force $F_{p c}$ and torque $Q_{p c}$ to restrict the deflection based on the rotating axis and angle $\theta$ :

$$
F_{p c}=m(d) *\left(\boldsymbol{P} \boldsymbol{X}_{i}-\frac{\boldsymbol{P} \boldsymbol{X}_{p}}{\left\|\boldsymbol{P} \boldsymbol{X}_{p}\right\|}\left\|\boldsymbol{P} \boldsymbol{X}^{\prime}\right\|\right), \quad Q_{p c}=n(\theta) * \theta * \frac{\boldsymbol{P} \boldsymbol{X}^{\prime} \times \boldsymbol{P} \boldsymbol{X}_{\boldsymbol{i}}}{\left\|\boldsymbol{P} \boldsymbol{X}^{\prime} \times \boldsymbol{P} \boldsymbol{X}_{\boldsymbol{i}}\right\|}
$$

where $m(d)$ is inversely proportional to the distance $d$ between $X_{i}$ and $X^{\prime}$ and $n(\theta)$ is inversely proportional to $\theta$.

Force Composition. In addition to the feedback forces along the needle and the path constraint force/torque, we have to add a device weight compensation 
force $\left(F_{w c}\right)$ and torque $\left(Q_{w c}\right)$ to the total force $F_{\text {total }}$ and total torque $Q_{\text {total }}$ respectively [9], since the structure and actuator mass of the haptic devices exert a downward force on the stylus tip even when no force is sent to the device. Consequently, the total force in every haptic frame is given by:

$$
F_{\text {total }}=\sum F_{\text {friction }}+F_{\text {pre-puncture }}+F_{\text {cutting }}+F_{p c}+F_{w c}, \quad Q_{\text {total }}=Q_{p c}+Q_{w c}
$$

where $\sum F_{\text {friction }}$ is the total friction force caused by all penetrated tissues; $F_{\text {pre-puncture }}$ is added during the puncture events and $F_{\text {cutting }}$ is added when the needle penetrates into liver; $F_{p c} / Q_{p c}$ and $F_{w c} / Q_{w c}$ are the path constraint force/torque added after skin penetration and the device weight compensation force/torque. All the force components can be calculated accordingly.

\section{Results and Discussion}

Our system is operated on machines with a configuration of $2.66 \mathrm{GHz}$ Intel P4 Dual Core CPU, 4GB of RAM, and an Nvidia FX8800 GPU. The overall system is shown in Fig. 4(a). Based on our client-server architecture supporting two haptic devices, the haptic frame-rate in both devices can be maintained over $1 \mathrm{kHz}$. The ultrasound volume being used in our system (as shown in Fig. 2(b)) is stitched from six ultrasound volumes acquired from a phantom (CIRS Model 057, mimicking human liver tissues) with different scan angles using a GE Voluson 730 ultrasound scanner. CT images are obtained on a GE Lightspeed 16-slice multidetector from the same phantom. The proposed 3D SIFT feature extraction and registration is less sensitive to the variance of rotation, luminance, and affine transformations. Compared to intensity based registration algorithm, our proposed 3D SIFT approach permits a highly automatic creation of ultrasound panorama with minimal manual work.

Several parameters in our haptic model have to be calibrated before starting the training procedure, including the elastic and viscous coefficients of involved

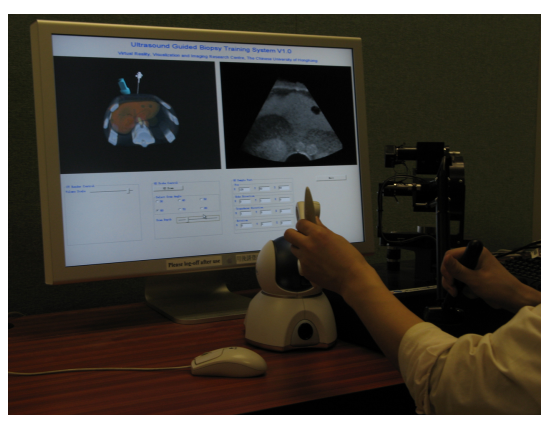

(a)

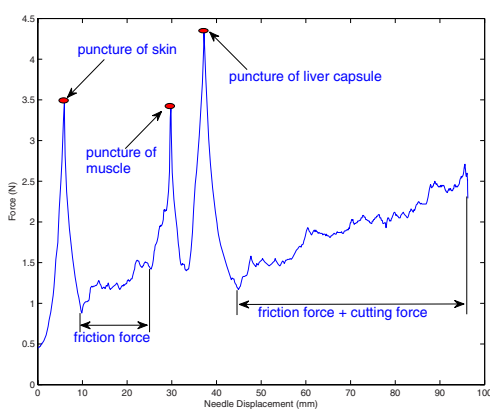

(b)

Fig. 4. (a) Overall system, and (b) force output obtained in a training session 
Table 1. The calibrated parameters of our force model

\begin{tabular}{|c||c|c|c|c|c|c|c|}
\hline Parameter & $k_{\text {skin }}$ & $\nu_{\text {skin }}$ & $\mu_{\text {skin }}$ & $\Delta x_{\text {skin }}$ & $\nu_{\text {ad }}$ & $\mu_{\text {ad }}$ & $k_{\text {muscle }}$ \\
\hline Values & $0.46 \mathrm{~N} / \mathrm{mm}$ & $0.02 \mathrm{Ns} / \mathrm{mm}$ & 0.18 & $0.1 \mathrm{~mm}$ & $0.01 \mathrm{Ns} / \mathrm{mm}$ & 0.1 & $0.19 \mathrm{~N} / \mathrm{mm}$ \\
\hline Parameter & $\nu_{\text {muscle }}$ & $\mu_{\text {muscle }}$ & $\Delta x_{\text {mucsle }}$ & $k_{\text {liver }}$ & $\nu_{\text {liver }}$ & $\mu_{\text {liver }}$ & $\Delta x_{\text {liver }}$ \\
\hline Values & $0.03 \mathrm{Ns} / \mathrm{mm}$ & 0.15 & $0.11 \mathrm{~mm}$ & $0.42 \mathrm{~N} / \mathrm{mm}$ & $0.02 \mathrm{Ns} / \mathrm{mm}$ & 0.58 & $0.12 \mathrm{~mm}$ \\
\hline
\end{tabular}

tissues, coefficients of kinetic friction of involved tissues, and the incremental displacement $\Delta x$ for the incremental viscoelastic model. In the initialization step, we set these parameters according to the experimental results in the biomechanical references mentioned previously. Parameters are fine adjusted based on the practical feelings of experienced surgeons. Results are shown in Table 1 In addition, we provide an interface to facilitate users in adaptively adjusting these parameters according to patient-specific biomechanical properties. We have invited 12 novices and 2 experienced radiologists to evaluate our system; users find our realistic visual and haptic renditions can improve the involvement and training of needle insertion. Fig. 4(b) shows the force-displacement relationship obtained in one complete needle insertion training session. It is clearly observed that three force peaks occur at the puncture of skin, muscle and liver's capsule. The resistance forces after puncturing liver are bigger than those after puncturing skin since we have introduced an extra force to simulate the cutting of interior structures in liver. It is demonstrated that our system can deliver users a realistic force feeling throughout the needle insertion procedure. Our system, based on the phantom, forms a good basis of US-guided biopsy training which can be naturally extended to real medical data, although more in-depth study of non-rigid US registration on patient-specific data have to be performed. Besides, we plan to introduce respiratory motion, needle bending or related deformations, as well as other US artifacts to our work in the future.

\section{Acknowledgment}

The work described in this paper was fully supported by a grant from the Research Grants Council of the Hong Kong Special Administrative Region (Project No. CUHK4461/05M). This work is also affiliated with the Virtual Reality, Visualization and Imaging Research Center at The Chinese University of Hong Kong as well as the Microsoft-CUHK Joint Laboratory for Human-Centric Computing and Interface Technologies.

\section{References}

1. Vidal, F.P., Chalmers, N., Gould, D.A., Healey, A.E., John, N.W.: Developing a needle guidance virtual environment with patient specific data and force feedback. In: Proc. of Computer Assisted Radiology and Surgery, pp. 418-423 (2005)

2. Magee, D., Zhu, Y., Ratnalingam, R., Gardner, P., Kessel, D.: An augmented reality simulator for ultrasound guided needle placement training. J. of Med. Bio. Eng. and Comput. 45(10), 957-967 (2007) 
3. Forest, C., Comas, O., Vaysière, C., Soler, L., Marescaux, J.: Ultrasound and needle insertion simulators built on real patient-based data. Stud. Health Technol. Inform. 125, 136-139 (2007)

4. Lowe, D.G.: Distinctive image features form scale-invariant keypoints. International Journal of Computer Vision 60(2), 91-110 (2004)

5. Scovanner, P., Ali, S., Shah, M.: A 3-dimensional sift descriptor and its application to action recognition. In: Proc. of Multimedia, pp. 357-360 (2007)

6. Hartley, R., Zisserman, A.: Multiple View Geometry in Computer Vision, 2nd edn. Cambridge University Press, Cambridge (2003)

7. Brett, P.N., Parker, T.J., Harrison, A.J., Thomas, T.A., Carr, A.: Simulation of resistance forces acting on surgical needles. J. of Eng. Med. 211(4), 335-347 (1997)

8. Okamura, A.M., Simone, C., O' Leary, M.D.: Force modeling for needle insertion into soft tissue. IEEE Trans. Bio. Eng. 51(10), 1707-1716 (2004)

9. Birtwisle, M., Bulpitt, A.: A 6 DOF gravity compensation scheme for a phantom premium using a neural network. Stud. Health Technol. Inform. 125, 43-48 (2007) 